\title{
Stability Analysis of Discrete-Time Takagi-Sugeno Fuzzy Systems
}

\author{
Renata Pytelková and Petr Hušek \\ Department of Control Engineering, Faculty of Electrical Engineering \\ Czech Technical University, \\ Technická 2, 16627 Praha 6, Czech Republic \\ \{pytelko, husek\}@control.felk.cvut.cz
}

\begin{abstract}
The paper presents a method analyzing asymptotic stability of discrete-time Takagi-Sugeno fuzzy systems. It is supposed that the plant is described by the Takagi-Sugeno fuzzy system with linear statespace submodels in the consequents of rules and the controller by the Takagi-Sugeno fuzzy system with linear state feedback submodels. These fuzzy systems can be considered as linear time-varying systems, where coefficients are varying in given intervals. The method tests stability by checking only products of vertex (extreme) matrices.
\end{abstract}

\section{Introduction}

Stability is one of the most important issues in analysis of control systems. In the case of fuzzy systems no particular stability theory exists. In fact the fuzzy system is a kind of nonlinear system with the disadvantage of having a complex analytical description.

There exist several particular methods designed for some special case of fuzzy systems. These methods are based on methods used in nonlinear control theory, for example small gain theorem and its related techniques (the circle criterion, conicity methods etc.), qualitative methods (stability indices) and the Lyapunov stability theory, among others. These methods usually put some constraints on the controller or to the model of the controlled system.

Special case of fuzzy systems are so-called Takagi-Sugeno fuzzy systems ([4]). In fuzzy control theory Takagi-Sugeno fuzzy systems with linear state-space or input-output submodels in the consequents of rules are used. The main advantage is, that this approach (sometimes called model based fuzzy approach) provides to combine together the knowledge of linear systems and fuzzy logic and use it for stability analysis. There exist two main approaches of stability analysis of such systems.

The first one is based on finding a common Lyapunov function for all subsystems used in consequents of rules. Problem of finding of this function is solved by LMI. This method states sufficient condition of stability and more details concerning this method can be found for example in [5]. However this algorithm is unable to decide about stability in many practical situations due to its big conservatism. 
Another approach can be found in [3]. In this paper it was shown, that problem of stability analysis of Takagi-Sugeno fuzzy systems with linear submodels in consequents of rules can be transformed to robust stability analysis of a polynomial with polynomic structure of its coefficients. Stability is tested by the Modified Jury or the Modified Routh criterion. It states sufficient and necessary stability conditions for slowly varying systems.

In this paper the new method for stability analysis of discrete-time TakagiSugeno fuzzy systems, where the plant is described by the Takagi-Sugeno fuzzy system with linear state-space submodels in the consequents of rules and the controller by the Takagi-Sugeno fuzzy system with linear state feedback submodels.

The paper is organized as follows. In section 2 the Takagi-Sugeno fuzzy model of a plant and the PDC controller is described. In section 3 the computation procedure of vertex extreme matrices of the closed-loop Takagi-Sugeno fuzzy system is introduced. Section 4 is devoted to the stability analysis method. Illustrative example is shown in the section 5 and some concluding remarks are mentioned in section 6 .

\section{Takagi-Sugeno Fuzzy Systems}

In this section Takagi-Sugeno fuzzy systems with linear submodels in the consequents of rules will be introduced. Linear state-space submodels are supposed.

The Takagi-Sugeno fuzzy system with the state-space submodels is consider in the following form:

$$
\begin{gathered}
R^{i}: \operatorname{IF~} z_{1}(t) \text { is } M_{1}^{i} \text { and } \ldots \text { and } z_{g}(t) \text { is } M_{g}^{i} \\
\operatorname{THEN} \mathbf{x}(t+1)=\mathbf{A}^{i} \mathbf{x}(t)+\mathbf{B}^{i} \mathbf{u}(t)
\end{gathered}
$$

for $i=1,2, \ldots, R$ where $\mathbf{z}(t)=\left[z_{1}(t), \ldots, z_{g}(t)\right]$ is a vector of variables of the premise (some measurable system variables), $M_{j}^{i}$ are fuzzy sets, $\mathbf{x}(t) \in \Re^{n}$ is the state vector, $\mathbf{u}(t) \in \Re^{m}$ is the input vector, $\mathbf{A}^{i} \in \Re^{n \times n}$ are linearized system matrices and $\mathbf{B}^{i} \in \Re^{n \times m}$ are linearized input matrices.

The total output of the fuzzy system is inferred as follows:

$$
\begin{aligned}
\mathbf{x}(t+1) & =\frac{\sum_{i=1}^{R} w_{i}(\mathbf{z}(t))\left\{\mathbf{A}^{i} \mathbf{x}(t)+\mathbf{B}^{i} \mathbf{u}(t)\right\}}{\sum_{i=1}^{R} w_{i}(\mathbf{z}(t))} \\
& =\sum_{i=1}^{R} h_{i}(\mathbf{z}(t))\left\{\mathbf{A}^{i} \mathbf{x}(t)+\mathbf{B}^{i} \mathbf{u}(t)\right\}
\end{aligned}
$$

where

$$
w_{i}(\mathbf{z}(t))=\prod_{j=1}^{g} M_{j}^{i}\left(z_{j}(t)\right)
$$


$M_{j}^{i}\left(z_{j}(t)\right)$ is the grade of membership of $z_{j}(t)$ in $M_{j}^{i}$. It is assumed that $w_{i}(\mathbf{z}(t)) \geq$ 0 , for $i=1,2, \ldots, R$ and $\sum_{i=1}^{R} w_{i}(\mathbf{z}(t))>0$ for all $t$. Therefore

$$
\sum_{i=1}^{R} h_{i}(\mathbf{z}(t))=1
$$

The PDC controller of the following form is considered:

$$
\begin{gathered}
R^{i}: \operatorname{IF~} z_{1}(t) \text { is } M_{1}^{i} \text { and } \ldots \text { and } z_{g}(t) \text { is } M_{g}^{i} \\
\operatorname{THEN} \mathbf{u}(t)=-\mathbf{K}^{i} \mathbf{x}(t)
\end{gathered}
$$

The total output of this fuzzy controller is

$$
\begin{aligned}
\mathbf{u}(t+1) & =\frac{-\sum_{i=1}^{R} w_{i}(\mathbf{z}(t)) \mathbf{K}^{i} \mathbf{x}(t)}{\sum_{i=1}^{R} w_{i}(\mathbf{z}(t))} \\
& =-\sum_{i=1}^{R} h_{i}(\mathbf{z}(t)) \mathbf{K}^{i} \mathbf{x}(t)
\end{aligned}
$$

By substituting (6) into (2) the total state space model of the fuzzy closed-loop system is obtained:

$$
\mathbf{x}(t+1)=\sum_{i=1}^{R} \sum_{j=1}^{R} h_{i}(\mathbf{z}(t)) h_{j}(\mathbf{z}(t))\left\{\mathbf{A}^{i}-\mathbf{B}^{i} \mathbf{K}^{j}\right\} \mathbf{x}(t)
$$

\section{Computation of Vertex Extreme Matrices}

The presented stability analysis method is based on stability analysis of linear time-varying systems, where coefficients are varying in given intervals. The method tests stability by checking only products of vertex extreme matrices.

Let's define the system matrix of the closed-loop system obtained from (7) as follows:

$$
\mathbf{G}(\mathbf{h}(\mathbf{z}(t)))=\sum_{i=1}^{R} \sum_{j=1}^{R} h_{i}(\mathbf{z}(t)) h_{j}(\mathbf{z}(t))\left\{\mathbf{A}^{i}-\mathbf{B}^{i} \mathbf{K}^{j}\right\}
$$

By substituting $h_{R}(\mathbf{z}(t))=1-\sum_{i=1}^{R-1} h_{i}(\mathbf{z}(t))$ into $(8), \mathbf{G}(\mathbf{h}(\mathbf{z}(t)))$ can be expressed as:

$$
\begin{aligned}
& \mathbf{G}(\mathbf{h}(.))=\sum_{i=1}^{R-1} \sum_{j=1}^{R-1} h_{i}(.) h_{j}(.)\left(\mathbf{B}^{j} \mathbf{K}^{R}+\mathbf{B}^{R} \mathbf{K}^{j}-\mathbf{B}^{i} \mathbf{K}^{j}-\mathbf{B}^{R} \mathbf{K}^{R}\right) \\
& +\sum_{j=1}^{R-1} h_{j}(.)\left(\mathbf{A}^{j}-\mathbf{B}^{j} \mathbf{K}^{R}-\mathbf{A}^{R}-\mathbf{B}^{R} \mathbf{K}^{j}+2 \mathbf{B}^{R} \mathbf{K}^{R}\right)+\mathbf{A}^{R}-\mathbf{B}^{R} \mathbf{K}^{R}
\end{aligned}
$$


Each element $g_{i j}(\mathbf{h}(\mathbf{z}(t)))$ of the matrix $\mathbf{G}(\mathbf{h}(\mathbf{z}(t)))$ is a polynomic function of the second order of the time-varying parameter $\mathbf{h}(\mathbf{z}(t))$ and can be rewritten to the following form:

$$
\begin{gathered}
g_{i j}(\mathbf{h})=\mathbf{h}^{T} \mathbf{C}^{(i j)} \mathbf{h}+\left(\mathbf{d}^{(i j)}\right)^{T} \mathbf{h}+v^{(i j)} \\
\mathbf{C}^{(i j)}=\left[c^{(i j)}\right] \in \Re^{R-1, R-1}, \mathbf{d}^{(i j)} \in \Re^{R-1}, v^{(i j)} \in \Re
\end{gathered}
$$

and

$$
\begin{gathered}
\mathbf{h} \in H \subset \Re^{R-1} \\
H=\left\{\mathbf{h} \in H \subset \Re^{R-1}: h_{k} \in[0,1], \sum_{k=1}^{R-1} h_{k} \leq 1, k=1, \ldots, R-1\right\}
\end{gathered}
$$

where $\mathbf{h}$ denotes $(\mathbf{h}(\mathbf{z}(t)))$.

The system matrix $\mathbf{G}(\mathbf{h})$ can be overbounded by the interval matrix $\mathbf{G}^{I}$ in the following form

$$
\mathbf{G}^{I}=\left[\begin{array}{ccc}
{\left[g_{11}^{\min }, g_{11}^{\max }\right]} & \cdots & {\left[g_{1 n}^{\min }, g_{1 n}^{\max }\right]} \\
\vdots & & \vdots \\
{\left[g_{n 1}^{\min }, g_{n 1}^{\max }\right]} & \cdots & {\left[g_{n n}^{\min }, g_{n n}^{\max }\right]}
\end{array}\right]
$$

The problem of finding extreme values of $g_{i j}(\mathbf{h})$ on a box $H$ is a task of mathematical programming ([1]). General formulation of a task of mathematical programming is as follows.

Let us consider the problem of minimization of a function $f_{0}(\mathbf{x})$, where the constraints are given in the form of inequalities

$$
\min \left\{f_{0}(\mathbf{x}) \mid f_{j}(\mathbf{x}) \leq b_{j}, j=1, \ldots, m\right\}
$$

Necessary conditions of extreme values can be determined by the following theorem.

Definition 1. Let a point ${ }^{0} \mathbf{x}$ satisfy all constraints of $(13)$. Let $J\left({ }^{0} \mathbf{x}\right)$ be the set of indices, for which the corresponding constraints are active (e.g. inequality changes to equality):

$$
J\left({ }^{0} \mathbf{x}\right)=\left\{j \mid f_{j}\left({ }^{0} \mathbf{x}\right)=b_{j}\right\}
$$

The point ${ }^{0} \mathbf{x}$ is said to be a regular point of the set $X$ given by constraints in (13), if the gradients $\nabla f_{j}\left({ }^{0} \mathbf{x}\right)$ are linearly independent $\forall j \in J\left({ }^{0} \mathbf{x}\right)$.

Theorem 1 (Kuhn-Tucker). Let ${ }^{*} \mathbf{x}$ be a regular point of a set $X$ and a function $f_{0}(\mathbf{x})$ has in some neighbourhood of ${ }^{*} \mathbf{x}$ continuous first partial derivatives. If the function $f_{0}(\mathbf{x})$ has in the point ${ }^{*} \mathbf{x}$ the local minimum on $X$, then there exists a (Lagrange) vector ${ }^{*} \lambda \in \Re^{m}$ that

$$
\begin{aligned}
\nabla f_{0}\left({ }^{*} \mathbf{x}\right)+\sum_{r=1}^{m}{ }^{*} \lambda_{r} \nabla f_{r}\left({ }^{*} \mathbf{x}\right) & =0 \\
{ }^{*} \lambda_{j}\left(f_{j}\left({ }^{*} \mathbf{x}\right)-b_{j}\right) & =0 \\
{ }^{*} \lambda_{j} & \geq 0
\end{aligned}
$$

hold $\forall j=1, \ldots, m$. 
Remark 1. For maximization of a function $f_{0}(\mathbf{x})$ the last inequality of (15) is replaced by ${ }^{*} \lambda_{j} \leq 0$.

To apply Theorem 1 for solving our problem it is necessary to check whether the preconditions of this theorem are satisfied. As $g_{i j}(\mathbf{h})$ is a polynomic function of the second order, its first partial derivatives are continuous $\forall \mathbf{h} \in H$ and the second assumption is satisfied. In our case

$$
\begin{aligned}
f_{0}(\mathbf{h}) & =g_{i j}(\mathbf{h}) \\
f_{j}(\mathbf{h}) & =(-1)^{j+1} h_{i} \\
b_{j} & =0 \text { for } j \text { even } \\
b_{j} & =1 \text { for } j \text { odd } \\
& R-1 \\
f_{2 R-1}(\mathbf{h}) & =\sum_{k=1}^{R} h_{k} \\
b_{2 R-1} & =1
\end{aligned}
$$

where $i=1, \ldots, R-1, j=1, \ldots, 2(R-1), j=2 i-1,2 i$.

Then

$$
\begin{gathered}
\nabla f_{j}(\mathbf{h})=(-1)^{j+1} \mathbf{e}^{(i)}, \forall \mathbf{h} \in H, j=1, \ldots, 2(R-1) \\
i=\frac{j+1}{2} \text { for } j \text { odd } i=\frac{j}{2} \text { for } j \text { even } \\
\nabla f_{2 R-1}(\mathbf{h})=[1, \ldots, 1]^{T}
\end{gathered}
$$

where $e^{(i)}=[0, \ldots, 0,1,0, \ldots, 0]^{T}$ with 1 on the $i$-th position. Because for some $\mathbf{h} \in H$ only even or only odd constraints (or none of them) can be active $\forall i=$ $1, \ldots, R-1, \nabla f_{j}(\mathbf{h})$, are linearly independent $\forall \mathbf{h} \in H, j \in J(\mathbf{h})$. It means that all points $\mathbf{h} \in H$ are regular ones.

According to Theorem 1 it is necessary to determine the gradient $\nabla g_{i j}(\mathbf{h})$. The components are

$$
\nabla g_{i j}(\mathbf{h})=\left[\begin{array}{lll}
\frac{\partial g_{i j}(\mathbf{h})}{\partial h_{1}} & \ldots & \frac{\partial g_{i j}(\mathbf{h})}{\partial h_{R-1}}
\end{array}\right]^{T}
$$

follows from (10)

$$
\begin{aligned}
& \frac{\partial g_{i j}(\mathbf{h})}{\partial h_{l}}=2 c_{l l}^{(i j)} \cdot h_{l}+\sum_{\substack{r=1 \\
r \neq l}}^{R-1}\left(c_{l r}^{(i j)}+c_{r l}^{(i j)}\right) \cdot h_{r}+d_{l}^{(i j)} \\
& i, j, l=1, \ldots, R-1
\end{aligned}
$$

After substituting (16), (17), (18) and (19) to (15) the following system of equations and inequalities is obtained:

$$
\left[\begin{array}{cccc}
2 c_{11}^{(i j)} & c_{12}^{(i j)}+c_{21}^{(i j)} & \cdots & c_{1, R-1}^{(i j)}+c_{R-1,1}^{(i j)} \\
c_{21}^{(i j)}+c_{12}^{(i j)} & 2 c_{22}^{(i j)} & \cdots & c_{2, R-1}^{(i j)}+c_{R-1,2}^{(i j)} \\
\vdots & \vdots & \ddots & \vdots \\
c_{R-1,1}^{(i j)}+c_{1, R-1}^{(i j)} & c_{R-1,2}^{(i j)}+c_{2, R-1}^{(i j)} & \cdots & 2 c_{R-1, R-1}^{(i j)}
\end{array}\right] \cdot\left[\begin{array}{c}
h_{1} \\
h_{2} \\
\vdots \\
h_{R-1}
\end{array}\right]+
$$




$$
\begin{aligned}
& +\left[\begin{array}{ccccccccc}
1 & -1 & 0 & 0 & 0 & \cdots & 0 & 0 & 1 \\
0 & 0 & 1 & -1 & 0 & \cdots & 0 & 0 & 1 \\
\vdots & \vdots & \vdots & \vdots & \vdots & \ddots & \vdots & \vdots & \vdots \\
0 & 0 & 0 & 0 & 0 & \cdots & 1 & -1 & 1
\end{array}\right] \cdot\left[\begin{array}{c}
\lambda_{1} \\
\lambda_{2} \\
\vdots \\
\lambda_{2 R-1}
\end{array}\right]=\left[\begin{array}{c}
d_{1}^{(i j)} \\
d_{2}^{(i j)} \\
\vdots \\
d_{R-1}^{(i j)}
\end{array}\right] \\
& \lambda_{1}\left(h_{1}-1\right)=0 \\
& \lambda_{2} h_{1}=0 \\
& \lambda_{3}\left(h_{2}-1\right)=0 \\
& \lambda_{4} h_{2}=0 \\
& \lambda_{2 \cdot R-3}\left(h_{R-1}-1\right)=0 \\
& \lambda_{2 \cdot(R-1)} h_{R-1}=0 \\
& \lambda_{2 \cdot R-1}\left(\sum_{k=1}^{R-1} h_{k}-1\right)=0
\end{aligned}
$$

$\lambda_{1}, \ldots, \lambda_{2 \cdot R-1} \geq 0$ for minimization

$\lambda_{1}, \ldots, \lambda_{2 \cdot R-1} \leq 0$ for maximization

The important fact is that the equation (20) is linear. The computational procedure of solving (20), (21) runs as follows. At first all solutions of (21) (nonlinear) are determined. This corresponds to determining of all the parts of the box $H$ - the interior and all the parts of the boundary of $H$ (all manifolds with the dimension $i, i=0, \ldots, R-2$ containing only points on the boundary of $H$ ). Each solution of (21) corresponds to $2 R-1$ linear equations (from (21) it follows that at least one of $\lambda_{2 i-1}, \lambda_{2 i} \forall i=1, \ldots, R-1$ has to equal zero; if $\lambda_{2 i-1}=0$ then either $\lambda_{2 i}=0$ or $h_{i}=0$, if $\lambda_{2 i}=0$ then either $\lambda_{2 i-1}=0$ or $h_{i}=1, \forall i=1, \ldots, R-1 ; \lambda_{2 R-1}$ can be zero anytime). These $2 R-1$ equations together with $R-1$ equations of (20) form $3 R-2$ linearly independent linear equations for $3 R-2$ unknown variables. It means that there exists a unique solution $\left({ }^{*} \lambda,{ }^{*} \mathbf{h}\right)$ (for each solution of $\left.(21)\right)$ of $(20)$ and $(21)$. As the number of manifolds with the dimension $i, i=0, \ldots, R-1$ containing only points on the boundary of $H$ is $\left(\begin{array}{c}R \\ i\end{array}\right)$, the total number $n_{s}$ of all solutions of (20) and (21) is given by

$$
n_{s}=\sum_{i=0}^{R-1}\left(\begin{array}{c}
R \\
i
\end{array}\right)=2^{R}-1
$$

In the next step is checked whether ${ }^{*} \lambda_{j}^{(l)} \geq 0\left({ }^{*} \lambda_{j}^{(l)} \leq 0\right), \forall j=1, \ldots, 2(R-1)$ and ${ }^{*} \mathbf{h}^{(l)} \in H$ for each $l=1, \ldots, n_{s}$. Denote by $L_{\min }\left(L_{\max }\right)$ the set of $l$ for which these conditions are satisfied.

$$
\begin{aligned}
& L_{\min }=\left\{l:{ }^{*} \mathbf{h}^{(l)} \in H,{ }^{*} \lambda_{j}^{(l)} \geq 0, \forall j=1, \ldots, 2(R-1)\right\} \\
& L_{\max }=\left\{l:{ }^{*} \mathbf{h}^{(l)} \in H,{ }^{*} \lambda_{j}^{(l)} \leq 0, \forall j=1, \ldots, 2(R-1)\right\}
\end{aligned}
$$


Then

$$
\begin{aligned}
g_{i j}^{\min }(\mathbf{h}) & =\min _{l \in L_{\min }}\left(g_{i j}\left({ }^{*} \mathbf{h}^{(l)}\right)\right) \\
g_{i j}^{\max }(\mathbf{h}) & =\max _{l \in L_{\max }}\left(g_{i j}\left({ }^{*} \mathbf{h}^{(l)}\right)\right)
\end{aligned}
$$

Now let's define the set of extreme matrices $\mathbf{G}^{E} \subset \mathbf{G}^{I}$ such that

$$
\mathbf{G}^{E}=\left\{\left[g_{i j}^{E}\right] \mid g_{i j}^{E}=g_{i j}^{\min } \text { or } g_{i j}^{\max } ; i, j=1, \ldots, R\right\}
$$

Obviously number of elements of $\mathbf{G}^{E}$ is $2^{p}$, where $p=n^{2}$ element of $\mathbf{G}^{E}$ will be refered as $\mathbf{G}_{j}^{E}$ with $j \in\left\{1, \ldots, 2^{p}\right\}$.

Let denote the set of all products of extreme matrices of length $k$ by

$$
\overline{\mathbf{P}}_{k}^{E}=\left\{\mathbf{P}_{k}^{E}\left(i_{1}, \ldots, i_{k}\right)=\mathbf{G}_{i_{1}}^{E} \cdot \ldots \cdot \mathbf{G}_{i_{k}}^{E} \mid\left(i_{1}, \ldots, i_{k}\right) \in\left\{1, \ldots, 2^{p}\right\}^{k}\right\}
$$

where $\left\{1, \ldots, 2^{p}\right\}^{k}$ is the $k$-th cross product of $\left\{1, \ldots, 2^{p}\right\}$. Therefore the number of elements of $\overline{\mathbf{P}}_{k}^{E}$ is $2^{p k}$.

\section{Stability Analysis}

Let us define $([2])$ the following theorem and lemma:

Theorem 2. $\left\|\prod_{n=1}^{k} \mathbf{G}(n)\right\|<1$ for all choices of $\mathbf{G}(n) \in \mathbf{G}^{I}, n=1, \ldots, k$, iff $\left\|\mathbf{P}_{k}^{E}\left(i_{1}, \ldots, i_{k}\right)\right\|<1$ for all $\mathbf{P}_{k}^{E}\left(i_{1}, \ldots, i_{k}\right) \in \overline{\mathbf{P}}_{k}^{E}$.

The symbol || || denotes the induced matrix 1-norm or the $\infty$-norm.

Lemma 1. Let $\mathbf{G}(n) \in \mathbf{G}^{I}$. Then the time-variant Takagi-Sugeno fuzzy system (7) is asymptotically stable if there exists a finite $k$ such that $\left\|\prod_{n=1}^{k} \mathbf{G}(n)\right\|<$ $1, \forall \mathbf{G}(n) \in \mathbf{G}^{I}, n=1, \ldots, k$.

The theorem together with the lemma can be used to formulate the following corollary:

Corollary 1. The time-variant Takagi-Sugeno fuzzy system (7) is asymptotically stable if there exists a finite $k$ such that $\left\|\mathbf{P}_{k}^{E}\left(i_{1}, \ldots, i_{k}\right)\right\|<1, \forall\left(i_{1}, \ldots, i_{k}\right) \in$ $\left\{1, \ldots, 2^{p}\right\}^{k}$

Proofs of the theorem, lemma and corollary can be found in [2].

\section{Stability test algorithm}

1. Compute the interval matrix $\mathbf{G}^{I}$

2. Set $k=1$, compute the set of extreme matrices $\mathbf{G}^{E}$

3. Generate all $2^{p k}$ products $\mathbf{P}_{k}^{E}\left(i_{1}, \ldots, i_{k}\right)$ and compute the norm of all products.

4. If all norms are smaller than 1 , the system is stable. In other case set $k=k+1$ and repeat step 3 . 


\section{Example}

Define the Takagi-Sugeno fuzzy system described by two following rules:

$$
\begin{aligned}
& R^{1}: \operatorname{IF} x_{1}(t) \text { is } M_{1}^{1} \operatorname{THEN} \mathbf{x}(t+1)=\mathbf{A}^{1} \mathbf{x}(t)+\mathbf{B}^{1} \mathbf{u}(t) \\
& R^{2}: \operatorname{IF~} x_{1}(t) \text { is } M_{1}^{2} \operatorname{THEN~} \mathbf{x}(t+1)=\mathbf{A}^{2} \mathbf{x}(t)+\mathbf{B}^{2} \mathbf{u}(t)
\end{aligned}
$$

and define controller described by the following rules:

$$
\begin{aligned}
& R^{1}: \text { IF } x_{1}(t) \text { is } M_{1}^{1} \text { THEN } \mathbf{u}(t)=\mathbf{K}^{1} \mathbf{x}(t) \\
& R^{2}: \text { IF } x_{1}(t) \text { is } M_{1}^{2} \text { THEN } \mathbf{u}(t)=\mathbf{K}^{2} \mathbf{x}(t)
\end{aligned}
$$

where

$$
\begin{gathered}
\mathbf{A}^{1}=\left[\begin{array}{cc}
-0.2 & 0.4 \\
0.5 & 0.5
\end{array}\right] \mathbf{B}^{1}=\left[\begin{array}{l}
1 \\
1
\end{array}\right] \mathbf{K}^{1}=\left[\begin{array}{ll}
0.4 & 0.3
\end{array}\right] \\
\mathbf{A}^{2}=\left[\begin{array}{cc}
-0.4 & 0.2 \\
0 & 0.5
\end{array}\right] \mathbf{B}^{2}=\left[\begin{array}{l}
1 \\
1
\end{array}\right] \mathbf{K}^{2}=\left[\begin{array}{ll}
-0.2 & 0.1
\end{array}\right]
\end{gathered}
$$

The interval matrix $\mathbf{G}^{I}$ is

$$
\mathbf{G}^{I}=\left[\begin{array}{cc}
{[-0.6,-0.2]} & {[0.1,0.1]} \\
{[0.1,0.2]} & {[0.2,0.4]}
\end{array}\right]
$$

In the following table the extreme product norms are shown:

Table 1. Extreme product norms

$$
\begin{array}{c|c|c}
k & \max \|\cdot\|_{1} & \max \|\cdot\|_{\infty} \\
\hline 1 & 0.47 & 0.42
\end{array}
$$

Table 1 shows that the Takagi-Sugeno system is stable.

\section{Conclusions}

In the paper a new method for stability analysis of discrete-time Takagi-Sugeno fuzzy systems is presented. Linear state space submodels are supposed in rule consequents of the model of the plant and the controller is described by the linear state feedback submodels (so called PDC controller). The method states a sufficient condition of stability and it is based on the stability analysis of time-varying systems, where the system matrix is varying in given interval. This condition allows to test stability by checking only products of extreme matrices. This condition is simple to implement, but the computational complexity increases exponentially with the the dimension $n$. 


\section{Acknowledgements}

This work has been supported by the research program No. J04/98:212300013 "Decision Making and Control for Manufacturing" of the Czech Technical University in Prague (sponsored by the Ministry of Education of the Czech Republic).

\section{References}

1. Kuhn H. W., A. Tucker.: Nonlinear Programming, in Proceedings of the 2nd Berkeley Symposium on Mathematical Statistics and Probability, University of California Press, Berkeley, (1951)

2. Bauer P.H., K. Premaratne, J. Durán (1993).: A Necessary and Sufficient Condition for Robust Asymptotic Stability of Time-Variand Discrete Systems, IEEE Transactions on Automatic Control, Vol. 38, No. 9 (1993) 1427-1430

3. Pytelková R., P. Hušek.: Stability of Slowly Varying Takagi-Sugeno Fuzzy Systems, in Proceedings of IFSA/NAFIPS World Congress, Vancouver, Canada, (2001)

4. Takagi T., M. Sugeno.: Fuzzy Identification of System and Its Applications to Modelling and Control, IEEE Transactions on Systems, Man \& Cybernetics, Vol. 15 (1985) 116-132

5. Tanaka K., T. Ikeda, H.O. Wang.: Robust Stabilization of a Class of Uncertain Nonlinear Systems via Fuzzy Control: Quadratic Stabilizability, $H_{\infty}$ Control Theory, and Linear Matrix Inequalities, IEEE Transactions on Fuzzy Systems, Vol. 4, No. 1 (1996) 1-13.

6. Hušek, R. Dvořáková.: Robust Stability of Polynomials with Polynomic Structure of Coefficients, in Proceedings of 8th IEEE Mediterranean Conference on Control \& Automation, Patras, Greece, (2000) 